\title{
A simple scattering model for measuring particle mass fractions in multiphase flows
}

\author{
Johan Carlson *, Pär-Erik Martinsson \\ EISLAB, Lulea University of Technology, SE-971 87 Luleå, Sweden
}

Accepted 15 March 2002

\begin{abstract}
In this paper we present a simple theoretical model of how pulsed ultrasound is attenuated by the particles in a solid/liquid flow. The theoretical model is then used to predict the attenuation of sound, given the mass fraction, the density, and the size distribution of the solid particles.

The model is verified experimentally for suspensions of $0-10 \%$ (by mass) Dolomite $\left((\mathrm{Ca}, \mathrm{Mg}) \mathrm{CO}_{3}\right)$ particles and water.

The experimental results show that the attenuation of sound due to particles varies linearly with mass fraction, and that the proposed theoretical model can be used to predict this attenuation. In all experiments the transmitter and receiver array were clamped onto the pipe wall, thus providing a completely non-invasive and non-intrusive measurement technique. (C) 2002 Elsevier Science B.V. All rights reserved.
\end{abstract}

Keywords: Ultrasound; Flow measurement; Multiphase flow; Scattering

\section{Introduction}

Different types of multiphase flows are present in a wide range of industrial processes. One important example is the mining industry, where iron ore powder is transported using water. It is therefore of interest to measure different parameters of multiphase flows consisting of a bulk liquid and a minor phase of solid particles. There are many different approaches to this, and the books [1] and [2] give a good overview of current methods for the measurement of both one phase and multiphase flows.

Most of the existing multiphase flowmeters are designed to measure either the total volume flow or the total mass flow. There are also techniques available for measuring gas holdup in gas/liquid flow, but many of these techniques involve sampling of the flow in an obstructing manner [3].

In our research project, focus has been on developing an ultrasonic technique capable of measuring the mass fraction of solid particles in a solid/liquid flow. The idea

\footnotetext{
${ }^{*}$ Corresponding author.
}

is to combine such technique with some other method for measuring the bulk mass flow, and thus be able to extract the mass flow of the phases. It is desirable that any method for doing this, should also work for different types of solid particles, and different particle size distributions. In a previous paper [4] we presented experimental results of measuring the excess attenuation in suspensions of Magnetite (iron ore, $\mathrm{Fe}_{3} \mathrm{O}_{4}$ ), and Dolomite $\left((\mathrm{Ca}, \mathrm{Mg}) \mathrm{CO}_{3}\right)$ particles in water. In that paper it was shown experimentally that the excess attenuation of pulsed ultrasound with center frequency of $1.5 \mathrm{MHz}$, increases linearly with the particle mass fraction.

When sound propagates through an inhomogeneous medium, such as a suspension of particles and water, the transmitted sound wave is scattered by the particles. Because of this scattering, the transmitted wave will be attenuated. There are many models available to theoretically determine the attenuation due to scattering, like Allegra and Hawley [6], and Urick [7]. The Allegra and Hawley model is often referred to, but because of its computational complexity it is not suitable for implementation in a flow meter. The simplifications needed in order to implement the model in a practical system will introduce large model errors, which can be very hard to 
interpret from the final results. In this paper we instead use a very simple physical model of the scattering, where we assume that the particles are rigid spheres. We show with experiments that, for the frequencies and particle sizes investigated, this is a reasonable approximation. The attenuation of sound is also affected by other properties of the suspension, such as temperature, pressure, and salinity [8]. In the experiments presented in this paper, temperature and pressure are kept constant, and we focus on the effect of particle radius and particle mass fractions, since these are the most dominant effects in the application at hand.

In Section 2 we derive a theoretical expression for the coefficient of excess attenuation. The model is based on well-known theory and the purpose is to show that even a relatively simple model can be used to predict the behavior of a complex practical system. In Section 3 we show how an experimental value of the attenuation can be obtained from measured data. This is then used to verify the theoretical model. Section 4.2 contains an analysis of the uncertainties involved in determining the experimental value of the attenuation coefficient.

\section{Theory}

The goal with this section is to derive an expression for the attenuation of sound that passes through a suspension of small solid particles and water.

For a sound wave that travels in pure water we can consider the water to be lossless. If, however, an object such as a small particle obstructs the wave, the wave will lose energy, and the most dominant loss process is scattering. In a suspension with many particles the scattering will repeat itself many times. When the incoming plane wave interacts with a particle, the sound is scattered in all directions. Assume that all sound energy scattered away from the receiving transducer is lost. Then, if all particles are of equal size and they all have the same mechanical properties the energy loss, $\mathrm{d} \Pi$, caused by $N$ particles can be written as

$\mathrm{d} \Pi=-N \Pi_{\mathrm{bs}}$,

where $\Pi_{\mathrm{bs}}$ is the backscattered energy from each particle and $N$ is the number of particles in the path of the wave. In order to proceed we must determine the number of particles in the path of the wave, and the scattered energy from each particle.

The mass fraction, $c_{\mathrm{m}}$, of particles in the suspension is

$c_{\mathrm{m}}=\frac{N m_{\text {particle }}}{m_{\text {liquid }}+N m_{\text {particle }}} \approx \frac{N m_{\text {particle }}}{m_{\text {liquid }}}$,

where $m_{\text {particle }}$ is the mass of one particle and $m_{\text {liquid }}$ is the mass of the liquid phase of the suspension. For a control volume with geometry as shown in Fig. 1 having width

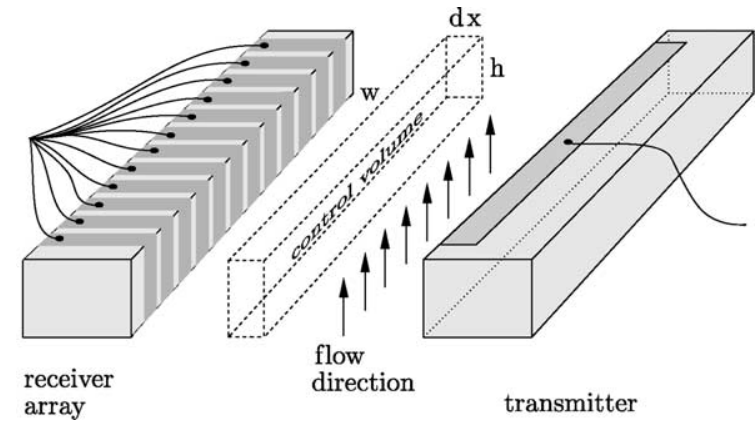

Fig. 1. Transmitter and receiver array used in the measurements, with notations used in the derivation of the attenuation coefficient.

$w$, height $h$, and length $\mathrm{d} x$ the mass of fluid inside the control volume is given by

$m_{\text {liquid }}=\rho_{1} w h \mathrm{~d} x$

and the mass of a spherical particle is

$m_{\text {particle }}=\rho_{\mathrm{p}} \frac{4}{3} \pi a^{3}$,

where $\rho_{1}$ and $\rho_{\mathrm{p}}$ is the densities of the liquid and the particles, respectively, and $a$ is the particle radius. From this we can estimate the number of particles as

$N \approx c_{\mathrm{m}} \frac{m_{\text {liquid }}}{m_{\text {particle }}}=c_{\mathrm{m}} \frac{3 \rho_{1} w h}{4 \rho_{\mathrm{p}} \pi a^{3}} \mathrm{~d} x$.

Now, when we have an estimate of the number of particles inside the control volume, we need to determine how each of these particles scatter the incoming sound wave. It should be noted, however, that if particles conglomerate, this will change the number of particles. It can be shown [9] that when a plane wave of intensity $I$ encounters an incompressible rigid sphere, the scattered intensity $I_{\mathrm{S}}$ is given by

$\frac{I_{\mathrm{s}}}{I}=\frac{16 \pi^{4} f^{4} a^{6}}{9 c^{4} r^{2}}(1-3 \cos \theta)^{2}$,

where $f$ is the frequency, $c$ is the speed of sound in the surrounding medium; $r$ and $\theta$ are the radius and angle coordinates of a polar coordinate system, respectively. This expression is valid for wavelengths such that $k a \ll 1$, where $k$ is the wave number. Fig. 2 shows the pattern of the scattered intensity. Even though Eq. (6) is given in polar coordinates $r$ and $\theta$ it is valid in three dimensions, because of symmetry around the $x$-axis. As expected and as Fig. 2 shows, most of the energy is backscattered, i.e. scattered in the opposite direction of the incoming sound wave. In fact only $1 / 8$ of the energy is scattered forward (to the right in Fig. 2). The same ratio is true as the long wavelength approximation holds $(k a \ll 1)$. The total backscattered power is found by integration of Eq. (6) over a half sphere with origin at the same position as the particle in question, that is 


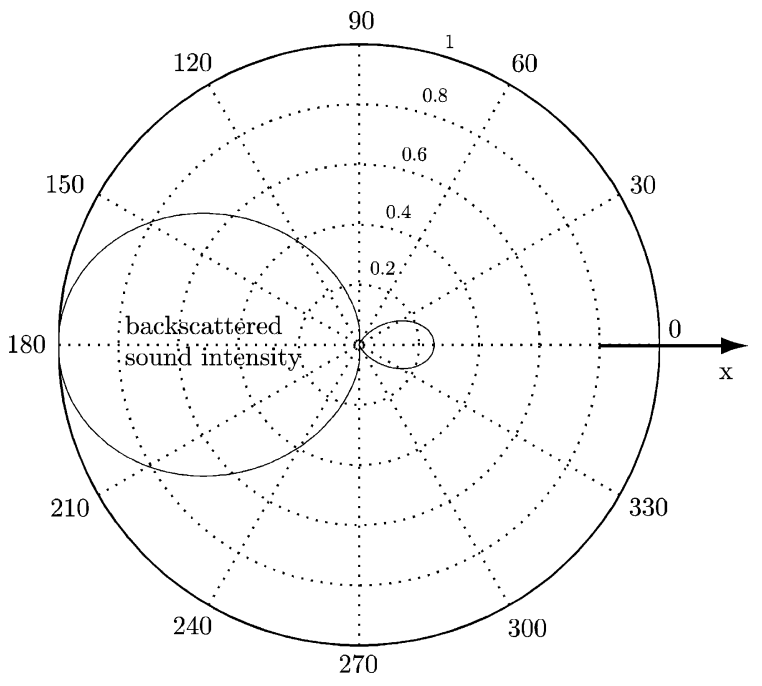

Fig. 2. Scattering from a rigid sphere when a plane wave is incident from the left in the picture $(k a=0.3)$.

$\Pi_{\mathrm{bs}}=\int_{\pi / 2}^{\pi} I_{\mathrm{s}}(r, \theta) 2 \pi r^{2} \sin \theta \mathrm{d} \theta=\frac{7}{8} \frac{256 \pi^{5} a^{6}}{9 \lambda^{4}} I$,

where the frequency has been replaced by the wavelength $\lambda=c / f$. For the control volume in Fig. 1 the intensity of the incoming wave is related to the energy by $I=\Pi / w h$, where $\Pi$ is the energy of the incoming plane wave, at position $x$. The backscattered energy now becomes

$\Pi_{\mathrm{bs}}=\frac{7}{8} \frac{256 \pi^{5} a^{6}}{9 \lambda^{4}} \frac{\Pi}{w h}$.

The use of Eq. (8) together with Eq. (5) in Eq. (1) leads to an ordinal differential equation for the energy loss of the sound wave:

$\frac{\mathrm{d} \Pi}{\Pi}=-A \frac{a^{3}}{\lambda^{4}} \frac{\rho_{1}}{\rho_{\mathrm{p}}} c_{\mathrm{m}} \mathrm{d} x$,

where the constant $A=168 \pi^{4} / 9$. Integration of both sides gives

$\int_{\Pi_{0}}^{\Pi} \frac{\mathrm{d} \widetilde{\Pi}}{\widetilde{\Pi}}=-\int_{0}^{x} A \frac{a^{3}}{\lambda^{4}} \frac{\rho_{1}}{\rho_{\mathrm{p}}} c_{\mathrm{m}} \mathrm{d} \tilde{x}$,

where $\widetilde{\Pi}$ and $\tilde{x}$ are integration variables, and $\Pi_{0}$ is transmitted energy at position $x=0$. After integration we have

$\frac{\Pi}{\Pi_{0}}=\mathrm{e}^{-c_{\mathrm{m}} \frac{a^{3} \rho_{1} \rho_{1}}{\lambda^{4} \rho_{\mathrm{p}}}}=\mathrm{e}^{-\alpha x}$,

where

$\alpha=c_{\mathrm{m}} A \frac{a^{3}}{\lambda^{4}} \frac{\rho_{1}}{\rho_{\mathrm{p}}}$

is the attenuation coefficient due to backscattering. As expected, the attenuation in Eq. (11) depends on the mass fraction of particles and the propagation distance of the wave. For a measurement system where the distance between the transducers is constant (see Fig. 1) the measured attenuation coefficient, $\alpha$, will be proportional to the mass fraction, $c_{\mathrm{m}}$, alone.

The expression for $\alpha$ in Eq. (12) is valid as long as the mass fraction of particles is low so that we can assume that there is no multiple scattering. Furthermore, in the model we assume a constant particle radius, $a$, and constant wavelength of the sound.

\section{Experiments}

The model derived in the previous section was verified with experiments. The following two subsections describe the experimental setup and the data analysis performed to experimentally determine the attenuation coefficient, $\alpha$, in Eq. (12).

\subsection{Measurements}

All measurements were made using a PVDF transmitter and a PVDF receiver array, see Fig. 1. The transmitter and receiver array were both made from a 28 $\mu \mathrm{m}$ thick PVDF sheet ${ }^{1}$, metallized with silver ink.

The receiver array consists of 12 elements, each $5 \mathrm{~mm}$ wide and $20 \mathrm{~mm}$ long (along the flow direction). The spacing between the receiver elements is about $1.5 \mathrm{~mm}$. The receiver was originally divided into several elements to be able to measure the attenuation of sound over a cross-section of the flow [4]. However, for the purpose of measuring only the mass fraction, the received energy is averaged over all receiver elements. This reduces the uncertainty of the measurements, in a similar way as using one big receiver would do.

The transmitter and receiver array were clamped onto the flow system depicted in Fig. 3. The transmitter and receiver array were mounted perpendicular to the flow direction. The purpose of the temperature controller is to keep the temperature constant during the experiment, since the attenuation of sound is strongly affected by temperature changes.

The pulses were sampled at $100 \mathrm{MHz}$ using a Nicolet 460 digitizing oscilloscope. The center frequency of the transmitted pulse is $1.5 \mathrm{MHz}$. The bandwidth of the pulse ranges from around $800 \mathrm{kHz}$ to $2 \mathrm{MHz}$, which is fairly narrowband given the characteristics of the receiving transducers. The bandwidth was measured by looking at the frequency where the spectrum of the pulse dropped by $3 \mathrm{~dB}$.

\footnotetext{
${ }^{1}$ The film PVDF film used in the measurements was manufactured by Measurement Specialties, Inc, Norristown, PA.
} 


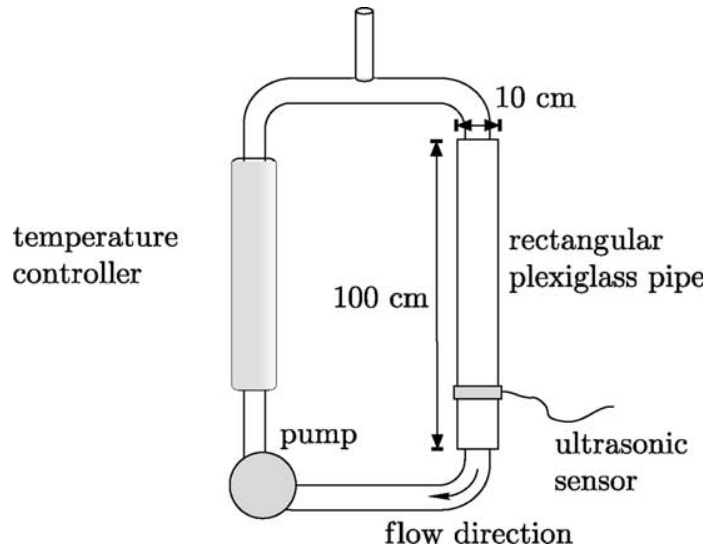

Fig. 3. Flow system used in the measurements.

\subsection{Data analysis}

Given the digitized ultrasound pulses we now show how the experimental value of the excess attenuation coefficient $\alpha$ from Eq. (12) can be obtained. Assuming the transmitted pulse is narrowband compared to the impulse response of the receiver elements, it can be shown that the same procedure as we use to determine the attenuation coefficient also calibrates the sensor to compensate for the effect of pipe wall, differences between receiver elements, losses in pure water, etc. [5].

Following the steps in [5], the energy of a sampled ultrasound pulse, $p[k]$ is calculated as

$E=\frac{1}{K} \sum_{k=1}^{K} p^{2}[k]$

where $K$ is the number of samples used to represent the pulse. Now assume that the received energy depends on the particle mass fraction, $c_{\mathrm{m}}$, as

$\left.E\left(c_{\mathrm{m}}\right)=E_{0} \mathrm{e}^{-\alpha\left(c_{\mathrm{m}}\right.}\right)$,

where $E_{0}$ is the received energy if the flow is pure water and $c$ is the particle mass fraction. The coefficient of excess attenuation, $\alpha\left(c_{\mathrm{m}}\right)$, due to the presence of particles is then given by

$\alpha\left(c_{\mathrm{m}}\right)=-\ln \left(\frac{E\left(c_{\mathrm{m}}\right)}{E_{0}}\right)=-\ln \left(\frac{\sum_{k=1}^{K} p_{c}^{2}[k]}{\sum_{k=1}^{K} p_{0}^{2}[k]}\right)$,

where $p_{c}[k]$ is a sampled version of the received pulse for mass fraction $c_{\mathrm{m}}$ and $p_{0}[k]$ is the corresponding measurement in pure water.

Now we compare the ratio $\Pi / \Pi_{0}$ in Eq. (11), derived from the equations for a continuous wave, with $E(c) / E_{0}$ as a similar property for pulsed ultrasound.

\section{Results}

In this section we describe the experiments and compare the experimental results with the theoretical model derived in Section 2. We also evaluate the uncertainty of the experimental results.

\subsection{Mass fraction measurements}

To verify the theoretical model we prepared suspensions of Dolomite particles $\left((\mathrm{Ca}, \mathrm{Mg}) \mathrm{CO}_{3}\right)$ for a mass fraction range from 0 to $10 \%$.

The particle size distribution was measured using a CILAS 1064 instrument. Fig. 4 shows the estimated size distribution. We then calculated the mean value of the particle radius and used this as input to the model.

Fig. 5 shows the measured and theoretical attenuation coefficients. The theoretical attenuation coefficient was calculated using a wavelength, $\lambda=0.987 \mathrm{~mm}$, speed of sound, $c=1480 \mathrm{~m} / \mathrm{s}$, particle density $\rho_{\mathrm{p}}=2800 \mathrm{~kg} /$ $\mathrm{m}^{3}$, and particle radius $a=26 \mu \mathrm{m}$. The measurements agree well with the predicted result, except for higher mass fractions and in a small region close to the origin. The small deviation close to the origin has been observed in other measurements we have made, but we still

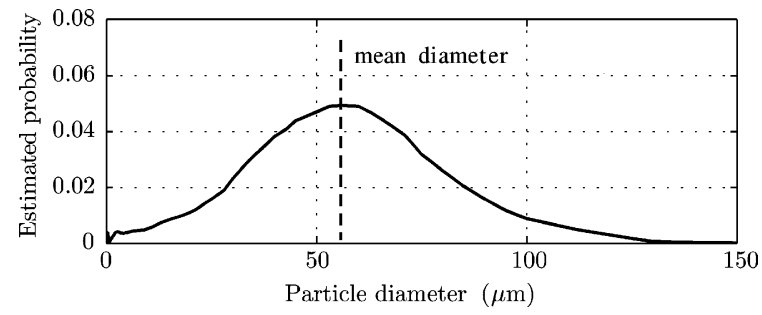

Fig. 4. Estimated size distribution of the Dolomite particles. The particle sizes were measured using a CILAS 1064 instrument.

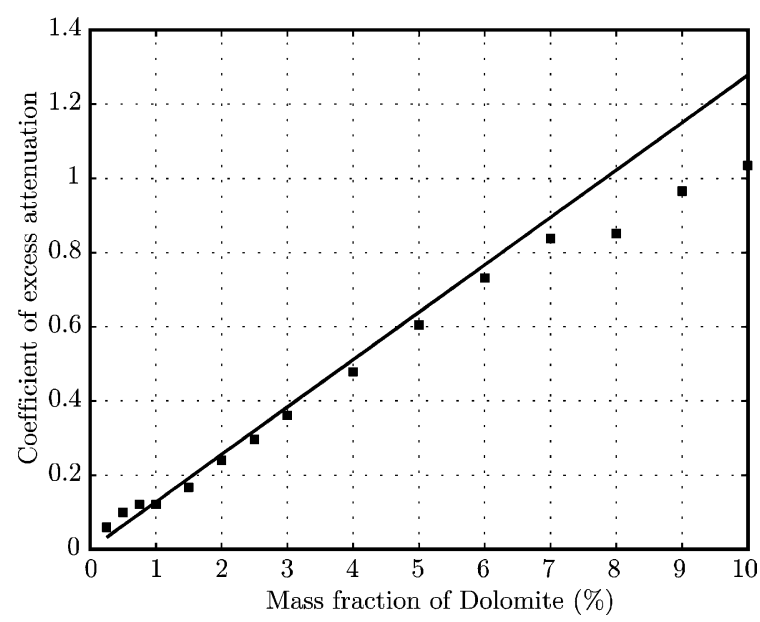

Fig. 5. Measured (dotted) and theoretical (solid) excess attenuation for Dolomite. 

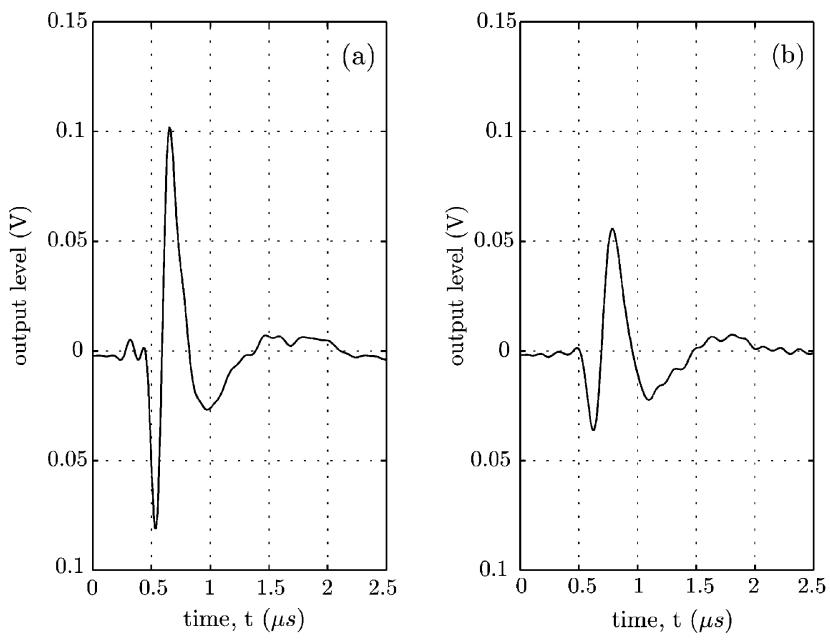

Fig. 6. Example of typical received pulses: (a) Received pulse for mass fraction $c_{\mathrm{m}}=2.5 \%$ and (b) received pulse for mass fraction $c_{\mathrm{m}}=9 \%$.

do not have any explanation to this. However, the deviations from the predicted results fall within the $95 \%$ confidence interval calculated in Section 4.2.

If there would be any multiple scattering of the sound waves for the higher mass fractions, the attenuation would increase faster, and not as the measurements show. The most probable explanation we have to this is that there is some sedimentation in the flow system because of the heavy particles. This causes particles to get stuck in cavities in the system. Another explanation is that when the particle mass fraction increases, the signal to noise ratio (SNR) decreases. The energy of the noise thus contributes more to the attenuation coefficient. This is illustrated by Fig. 6. We see that the amplitude of the received pulse is significantly lower for the higher mass fraction, while it is reasonable to believe that the noise variance remains unchanged.

We also made measurements for smaller particle diameters, and measured the attenuation, and for other types of solid particles. The measured attenuation coefficient still shows the same linear dependency of the mass fraction. However, the instrument we used to estimate particle diameters is not capable of measuring the smaller diameters, and therefore we are not able to compare these results with the theoretical model. The experimental results are shown in a previous paper [4].

\subsection{Uncertainty analysis}

Given the number of pulses measured at each receiver element and the standard deviation of their energies, we analyze the uncertainty of the mass fraction measurements. We also discuss other sources of error and the effect this might have on the final result.

For each of the 12 receiver elements, 150 pulses were measured for each particle mass fraction. Fig. 5 shows the average excess attenuation taken over all pulses at all receiver elements, that is

$\bar{\alpha}\left(c_{\mathrm{m}}\right)=\frac{1}{12 \times 150} \sum_{k=1}^{12} \sum_{l=1}^{150} \alpha_{k, l}\left(c_{\mathrm{m}}\right)$

where $\alpha_{k, l}(c)$ is the excess attenuation for pulse $l$ at receiver $k$ for mass fraction $c_{\mathrm{m}}$.

Assume that the coefficient of excess attenuation is a stochastic variable. If we assume that for each receiver element this stochastic variable belongs to the same distribution. Then, because of the central limit theorem, their average will be approximately normally distributed. If we average the attenuation coefficient over 150 measurements and over the 12 receiver elements, this average will be normally distributed with mean $\bar{\alpha}\left(c_{\mathrm{m}}\right)$ and variance $\bar{\sigma}^{2}$. Since the number of measurements is large, the resulting confidence interval can be calculated using the normal distribution [10].

The accuracy of the measurement method thus depends on how accurately we can determine $\bar{\alpha}\left(c_{\mathrm{m}}\right)$ in Eq. (16). A confidence interval for $\bar{\alpha}\left(c_{\mathrm{m}}\right)$ can be calculated from the estimated variance between different measurements, as

$\bar{\sigma}^{2}=\frac{1}{12} \sum_{k=1}^{12} \bar{\sigma}_{k}^{2}$

where $\bar{\sigma}_{k}^{2}$ is the variance of the measured average excess attenuation at receiver $k$.

From measured data we estimated the standard deviation for each of the mass fractions. The maximum value was found to be $\bar{\sigma}=0.0038$. The corresponding $95 \%$ confidence interval for the average excess attenuation is then $\bar{\alpha}(c) \pm 2 \bar{\sigma}=\bar{\alpha} \pm 0.0076$. This corresponds to a relative full scale error (at 7\% mass fraction) of approximately $\pm 1 \%$.

The analysis above does not include possible bias errors. For example, sedimentation of particles in cavities of the flow system will give rise to bias errors. Also, if particles conglomerate, this will lead to a higher attenuation, since the scattering cross-section will increase. In the model, however, we assume that the particle radius is equal to the average particle radius of the sample.

\section{Conclusions and discussion}

We have presented a simple theoretical model for the coefficient of excess attenuation, $\alpha$, of pulsed ultrasound due to the presence of particles. The model predicts that the attenuation coefficient varies linearly with particle mass fraction. This is also verified with experiments for suspensions of Dolomite $\left((\mathrm{Ca}, \mathrm{Mg}) \mathrm{CO}_{3}\right)$ particles for mass fractions in the range $0-10 \%$.

Because the model is a simple straight line relationship, this can easily be implemented in a system with 
very limited computational power. All model parameters can be determined a priori and thus, a sensor based on the presented model does not have to go through extensive calibration procedures in order to estimate the straight line. The only calibration needed is to measure the received ultrasound energy in a flow of pure water. This is then used to calibrate the sensor to compensate for reflections from pipe walls and for differences in transmitter and receiver characteristics.

In the model we assume that the particles can be regarded as rigid spheres. Judging by the experimental results this seems to be a reasonable approximation for the Dolomite particles used in the experiments. The effect of non-rigid particles is that their scattering crosssection is bigger than for the non-rigid case [9], which leads to a higher attenuation. Scattering by spherical particles is a subject that has been thoroughly studied by others and for more details the reader is referred to the work by Allegra and Hawley [6] and Epstein and Carhart [11].

Furthermore, in the model we assume constant particle radius and monochromatic sound waves, but in practice the particle size distribution is Gaussian-like. Also, the pulses have a certain bandwidth, and thus we have an interval of wavelengths rather than a single wavelength. In Eq. (12) we have the third power of the radius and the fourth power of the wavelength. Increasing either the radius or the frequency will increase the attenuation. However, incorporating this in the model, averaging over the particle size distribution and integrating over the spectrum, we found the resulting attenuation to be almost the same. From this we conclude that, for the frequencies and particle sizes examined here, these effects cancel out, and using the center frequency and the mean particle radius is a reasonable approximation.

One thing that remains to be investigated is the sensitivity of the method to variations in temperature. At the moment the temperature controller in Fig. 3 ensures that the temperature is kept constant during the measurements. We also need to investigate the sensitivity to installation effects such as pipe bends and pulsating flow. We know that flow system geometry affects the distribution of particles within the flow pipe [4], and that using an array of receivers or a large single-element receiver will reduce these effects. In the same paper we show that increasing the flow velocity from $2 \mathrm{~m} / \mathrm{s}$ to around $5 \mathrm{~m} / \mathrm{s}$, and by this introducing more turbulence, does not significantly affect the measured attenuation. In the experiments we noticed that if the signal to noise ratio decreases too much, the measured attenuation coefficient will not vary linearly with mass fraction. In the experiments presented in this paper we used a simple PVDF transducer. Changing this to a standard crystal transducer the SNR will increase significantly, thus extending the mass fraction interval that can be studied.

\section{Acknowledgements}

The authors would like to express their gratitude towards Prof. Bertil Pålsson for his help with determining the particle size distribution, and to Prof. Jerker Delsing and Prof. Anders Grennberg for valuable comments and discussions. Finally, the authors wishes to express their sincerest gratitude towards the reviewers for their valuable comments.

\section{References}

[1] J. Chaoki, L. Larachi, M.P. Dudoković, Non-Invasive Monitoring of Multiphase Flows, Elsevier, Amsterdam, 1997.

[2] D.W. Spitzer (Ed.), Flow Measurement-Practical Guides for Measurement and Control, Instrument Soc. Am., 1991.

[3] P. Mehdizadeh, D. Farchy, Multi-phase flow metering using dissimilar flow sensors: theory and field trial results, Proc. of SPE Middle East Oil Show, Bahrain, March 11-14, 1995, pp. 59-72.

[4] J. Carlson, Joint measurement of particle distribution and particle mass fraction in multiphase flows using a clamp-on PVDF array, Proc. of Flow Measurement 2001, Peebles, Scotland, UK, May $7-$ 10, 2001.

[5] J. Carlson, Ultrasonic Characterization of Materials and Multiphase Flows, Ph.D. Thesis, Luleå University of Technology, Luleå, Sweden, March 2002 (Chapter D).

[6] J.R. Allegra, S.A. Hawley, Attenuation of sound in suspensions and emulsions: theory and experiments, J. Acoustical Soc. Am. 51 (5) (1972) 1545-1564.

[7] R.J. Urick, The absorption of sound in suspensions of irregular particles, J. Acoustical Soc. Am. 20 (3) (1948) 283-289.

[8] S.D. Richards, The effect of temperature, pressure, and salinity on sound attenuation in turbid seawater, J. Acoustical Soc. Am. 103 (1) (1998) 205-211.

[9] P.M. Morse, K.U. Ingard, Theoretical Acoustics, Princeton University Press, Princeton, NJ, 1986.

[10] G.E.P. Box, W.G. Hunter, J.S. Hunter, Statistics for Experimenters, John Wiley and Sons, New York, 1978.

[11] P.S. Epstein, R.R. Carhart, The absorption of sound in suspension and emulsions. I. Water fog in air, J. Acoustical Soc. Am. 25 (1953) 553-565. 